\title{
Moving Beyond Cancer: Immediate Impact on the Health-Related Quality of Life of Breast Cancer Patients After Mastectomy
}

\author{
Rahman MM ${ }^{1}$, Hossain AFMA ${ }^{1}$, Ahsan $\mathrm{A}^{2}$, Monalisa NN³ ${ }^{3}$, Rahman $\mathrm{K}^{1}$, Azad SA \\ ${ }^{1}$ Department of Surgical Oncology, National Institute of Cancer Research and Hospital, Mohakhali, \\ Dhaka, ${ }^{2}$ Department of Medical Oncology, Shaheed Surwardy Medical College, Sherebanglanagar, \\ Dhaka, ${ }^{3}$ Centre for control of chronic diseases, icddrb, Mohakhali, Dhaka, ${ }^{4}$ National Institute of Cancer \\ Research and Hospital, Mohakhali, Dhaka. \\ Email: mizannicrh@gmail.com
}

\begin{abstract}
During the last decade, survival rates for breast cancer have increased as a result of earlier detection and increased use of adjuvant therapy. Limited data exist on the post mastectomy quality of life in the process of transition from health to cancer in its different phases of treatment. The aim of current study was to evaluate the changes of health-related quality of life (HRQoL) after mastectomy and to measure their impression about future perspectives in Bangladeshi Breasts cancer patients. A group of 250 women with a diagnosis of primary breast cancer who was admitted in the department of surgical oncology in National Institute of Cancer Research and Hospital, Dhaka were enrolled in this study from January 2012 to March 2013. Two-point interview like pre(M1) and post mastectomy(M2) was taken using the structured questionnaire made by European Organization for Research and Treatment of Cancer (EORTC)- EORTC Q30 and BR23. The mean age of the patients was $44.7 \pm 9.87$ years, $52.6 \%$ were locally advanced. HRQoL scores of breast cancer patients deteriorated after mastectomy, general features like presence of fatigability, nausea, apatite loss, sleeplessness and pain has got tremendous effect on the quality of life $(\mathrm{p}<.005)$. Financial difficulties and sleep disorders did not affect much. Four of the Global Health status/QOL parameters like physical functioning, role functioning, emotional functioning, cognitive functioning deteriorated but only physical functioning status affected significantly $(\mathrm{p}<0.005)$. Breast symptoms like body image problem, general breast symptoms like pain, arm swelling and sexual feeling affected their (HRQOL) after mastectomy. A diagnosis of cancer and associated treatments affects multiple domains of life. The complicated transitions between health, illness and living with cancer can often be challenged with a preoperative information for the patient confronted with breast cancer should include possible psychological effects of cancer diagnosis, surgery, and other treatment. Breast conservation particularly in the young group may give a better outcome in the treatment protocol of breast cancer patients.
\end{abstract}

Key words: Quality of Life, Breast Cancer, Moving beyond Cancer, Mastectomy

\section{Introduction}

Breast cancer is the most common cancer in women, and $86 \%$ of patients survive for at least five years after diagnosis, yielding more than two million women living with a history of the disease in the United States. ${ }^{1}$ Thus, preparation for favorable survivorship is a realizable goal for most women. The transition from patient to survivor, also termed the re-entry transition, ${ }^{2,3}$ is an understudied period. Once treatment ends, patients no longer interact frequently with the medical team and may lose the accompanying sense of protection. ${ }^{4}$ Cancer patients also often cite a downturn in emotional support at this time. They often must address fears about cancer recurrence and the pace at which persistent decrements in physical functioning will diminish. ${ }^{5,6}$ the most frequent concerns were fear of cancer recurrence, pain, death, harm from adjuvant treatment, and medical bills.

Over the last decades, the number of long-term survivors of breast cancer has increased because 
of advances in early diagnosis, as well as surgical and adjuvant treatments. Thus, long-term quality of life (QOL) and factors affecting QOL are of growing research interest. The QOL construct refers to a general sense of well-being in multiple dimensions of life. However, because of the variety of divergent definitions and measures of the construct results of QOL research in cancer patients are varied and often contradictory. ${ }^{7}$ Longitudinal studies about changes of QOL and persistent impairments in breast cancer patients show conflicting results. Furthermore, there are few data about long-term anxiety in women with breast cancer. ${ }^{8}$

Clinical practice reveals that long-term survivors are mostly distressed by anxiety, especially by fear of recurrence.

Research in the past decade mainly focused on possible demographic and clinical factors affecting QOL in breast cancer patients. Investigation of QOL in oncological subgroups is important for drawing conclusions about operation or therapy modalities. Most studies investigated single parameters, such as primary surgical treatment modality or age $e^{9,10}$ whereas other factors, such as tumor stage, were often neglected. There is a lack of studies investigating the impact of several oncological factors on long term QOL within the same clinical trial. ${ }^{8}$ Also, the impact of these factors on QOL is controversial.

However, limited data exist on the experiences of women during the critical transitional period between the end of primary treatment and survivorship the time when women must move beyond cancer to reestablish their normal life patterns. Having a better understanding of how patients navigate this transitional period is increasingly important, given the widespread use of adjuvant chemotherapy and the extension in time and complexity of primary treatments. ${ }^{11}$ For example, adjuvant chemotherapy and or radiotherapy treatment, whether given before or after surgery, often lasts more than six months. Consequently, the impact of contemporary primary treatment regimens on short-term quality of life (QOL) is largely unknown.

To address this period of transition, we conducted the five functional scales that evaluate physical functioning, role functioning, emotional functioning, cognitive functioning, and social functioning according to EORTC Q30. The additional module QLQ-BR23 contains breastcancer-specific scales like body image, arm symptoms, sexual functioning and breast symptoms. Our project covered "Moving Beyond Cancer "(MBC) Study after mastectomy to examine their different functional scales.

We report on the health status and QOL of women with breast cancer who had completed surgical treatment and are in the process of adjuvant treatment focusing on the above mentioned variables.

\section{Materials and Methods}

It is a prospective study. Patients cytologically or histologically diagnosed as primary breast carcinoma who attended to surgical oncology department, National Institute of Cancer Research and Hospital Dhaka from January 2012 to March 2013 for surgical treatment were included in this study. Regarding the Eligibility criteria having histopathologically proven breast cancer with stage I to Stage IV disease. Stage III and Stage IV diseased patient would have to be admitted after receiving neoadjuvant chemotherapy. One interview was taken before mastectomy in the hospital bed who were waiting for surgery after admission. Another interview was taken as outpatient basis after 4-6 weeks of mastectomy when they came for completion of chemotherapy or for adjuvant chemotherapy after satisfactory wound healing. Interview was taken by research assistants who are female medical graduates, having knowledge about breast cancer, research methodology, and previous experience of data collection. To address both the general and specific objectives the research carried out using a well-designed close type structured questionnaire made by European Organization for Research and Treatment of Cancer (EORTC). It was EORTC Q30 and BR23. We had to be registered and approved by the authority to use the Bangla Questionnaire supplied by Brussels based EORTC authority. The interview of the study participants was taken by giving them adequate time and privacy. Just after enrollment, first interview was taken. Before mastectomy patients were labeled as M1 and after mastectomy they were labeled as M2. A day long workshop and pre testing on questionnaire was arranged for the local research 
assistants for better understanding prior starting the project. The scoring formula supplied by EORTC was used for both the questionnaires compiled an analyzed with SPSS programme.

In this work, we focus on comparisons among health-related QOL outcomes, sexual functioning, and symptoms, and the four treatment groups (Prior to mastectomy with/without neoadjuvant chemotherapy, and mastectomy with/without neoadjuvant chemotherapy). In additional analyses, we investigated sexual functioning variables, including separate categorical measures of sexual limitations and composite summary scores, and variables reflecting potential treatment symptoms or menopausal symptoms that women reported. In addition, we used the test to implement analysis of covariance models controlling for demographic variables i.e., age, address, educational level, family income occupational status, marital status. Linear transformation was used to standardize the raw scores of the QOL parameters so that all scores ranged from 0 to 100 . In accordance with the scoring manual, the questionnaire items of the QLQ-C30 and the QLQ-BR23 were grouped into scales in the categories like Functional Scales and Symptom Scales. The additional items Cosmetic Confidence and Sexual Functioning were calculated according to the scoring manual of the QLQ-C30. We investigated the following oncological factors: patient's age at time of primary diagnosis. Patients' age was mediandichotomized into two categories: $\leq 40$ years and $>40$ years. We checked the distributions of scores at baseline: functional scales, body image, arm and breast symptoms, cosmetic confidence, sexual functioning, and the two anxiety scales were not normally distributed. All statistical tests were two-sided.

\section{Result}

Two hundred and fifty (250) women with breast cancer were included in the study. The mean was 44.7 years, standard deviation (SD) was 9.82 (range: 21-67). Family history of breast cancer was positive in $4 \%$ of patients. Mastectomy was done in all patients. There was no operative mortality.

Tumor profile was also investigated.it included size of the tumor, axillary lymph node status, metastasis and receptor status of the tumor.
Table I: show clinical profile of the patients

\begin{tabular}{lll}
\hline \multicolumn{1}{c}{ Factors } & $\begin{array}{l}\text { Frequency } \backslash \\
\text { Percentage }\end{array}$ \\
\hline Age $\leq 40$ years & $98(39.2)$ \\
40 years & $152(60.8)$ \\
Lump size & & \\
$<2 \mathrm{~cm}(\mathrm{~T} 1)$ & 5.9 & \\
$2.1-5 \mathrm{~cm}(\mathrm{~T} 2)$ & 40.7 & \\
$5.1-10 \mathrm{~cm}(\mathrm{~T} 3)>10 \mathrm{~cm}$ & 21.2 & \\
(T4) & 31.4 & \\
No lump & 0.8 & \\
Axillary lymph node & & \\
involvement No(No) & & \\
Mobile(N1) & 20 & \\
Fixed (N2) & 67.2 & \\
Supraclavicular (N3) & 7.2 & \\
Metastasis- & 5.6 & \\
& 16 & \\
Receptors (n=135) & $<40 \mathrm{yrs}$ of age & $>40 \mathrm{yrs}$ of age \\
ER+vePR+veHer2+ve & $18(30 \%)$ & $32(42.6 \%)$ \\
ER+ve PR+veHer2-ve & $9(15 \%)$ & $8(10.6 \%)$ \\
ER-ve PR-ve Her2+ve & $21(35 \%)$ & $15(20 \%)$ \\
ER-ve PR-ve Her2-ve & $12(20 \%)$ & $20(26.6 \%)$ \\
\hline
\end{tabular}

Clinical characters: Table I describes tumor profile of the patients: the mean age of the 250 participating patients mean was 44.7 years, majority $(>60 \%)$ was above 40 years, $52.6 \%$ patients were in the group of locally advanced breast cancer (T3 and T4). Axillary lymph node involvement was present in $80 \%$ of cases, metastasis (bony and visceral) was found in 16 cases, $61.6 \%$ patient received neoadjuvant chemotherapy. Receptor study $(n=135)$ showed that estrogen receptor was positive in 53.2\% cases among the elderly group ( $>40$ years). Over one fourth $(26.6 \%)$ of the elderly group were Triple negative breast cancer patients.

Health-related Quality of life: Quality of life was evaluated by the Quality of Life Questionnaire QLQ-C30, Version 3.0 of the EORTC Study Group on Quality of Life. ${ }^{12,13}$ The questionnaire is composed of the Global Health status/QOL Scale and five functional scales that evaluate physical functioning, role functioning, emotional functioning, cognitive functioning, and social functioning. Questionnaire in the physical functioning exclusively included her daily major and minor activities by herself. Higher mean scores on these scales represent better functioning and QOL. The additional module QLQ-BR23 contains breast-cancer-specific scales. Here, the three subscales: body image, arm symptoms, and breast symptoms, were used. Higher mean values 
on the arm- and breast-symptom scales indicate an increased extent of symptoms. Higher mean scores on the Body Image scale of the QLQ-BR23 and the additional scales for Cosmetic Confidence and Sexual Functioning represent better functioning.

Quality of life parameters: Health related quality of life was measured on two points along with breast related problems. They were categorized as premastectomy (M1) and post mastectomy (M2) groups subscales as neoadjuvant and no chemotherapy group of patients.

Table II: Showing Breast cancer patients' two point (M1 and M2) functioning and global quality of life scores as measured by the EORTC QLQ-C30* $(\mathrm{n}=250)$

\begin{tabular}{lccccc}
\hline \multicolumn{1}{c}{ Parameters } & $\begin{array}{l}\text { Mean } \\
\text { score } \\
\text { (M1) }\end{array}$ & SD & $\begin{array}{l}\text { Mean } \\
\text { score } \\
\text { (M2) }\end{array}$ & SD & $\mathrm{p}$ \\
\hline $\begin{array}{l}\text { Physical } \\
\text { functioning (PF2) }\end{array}$ & 83.58 & 10.01 & 64.30 & 4.002 & .028 \\
$\begin{array}{l}\text { Role functioning } \\
\text { (RF2) }\end{array}$ & 77.12 & 1.40 & 54.20 & 7.339 & .061 \\
$\begin{array}{l}\text { Emotional } \\
\text { functioning (EF) }\end{array}$ & 80.37 & 9.19 & 66.14 & 10.26 & .070 \\
$\begin{array}{l}\text { Cognitive } \\
\text { functioning(CF) }\end{array}$ & 84.69 & 1.9 & 77.30 & 10.40 & .060 \\
$\begin{array}{l}\text { Social functioning } \\
\text { (SF) }\end{array}$ & 38.81 & 1.2 & 49.03 & 6.89 & .063 \\
\hline
\end{tabular}

*The higher values indicate higher level of functioning and quality of life, min: $0, \max : 100$

Table III: showing Breast cancer patients' pre-operative and post-operative symptoms scores as measured by the EORTC QLQ-C30*(n=250)

\begin{tabular}{llllll}
\hline Parameters & $\begin{array}{l}\text { Mean } \\
\text { score } \\
\text { (M1) }\end{array}$ & SD & $\begin{array}{l}\text { Mean } \\
\text { score } \\
\text { (M2) }\end{array}$ & SD & $\mathrm{p}$ \\
\hline Fatigue(FA) & 68.48 & 8.6 & 64.69 & 7.89 & .05 \\
Nausea and & 86.62 & .76 & 68.67 & 11.68 & .07 \\
Vomiting(NV) & & & & & \\
Pain(PA) & 59.21 & 7.01 & 54.01 & 6.62 & .05 \\
$\begin{array}{l}\text { Dyspnea (DY) } \\
\text { Insomnia (SL) }\end{array}$ & 68.13 & 8.2 & 68.50 & 13.43 & .08 \\
$\begin{array}{l}\text { Appetite Loss } \\
\text { (AP) }\end{array}$ & 58.95 & 4.03 & 68.50 & 13.43 & .08 \\
$\begin{array}{l}\text { Financial } \\
\text { difficulties (F1) }\end{array}$ & 35.47 & .45 & 68.50 & 13.43 & .08 \\
\hline
\end{tabular}

*The higher values indicate a greater degree of symptoms, min: 0 , max: 100

Table II shows that physical functioning (PF) score was much higher in premastectomy group $(\mathrm{p}=0.028)$. Role functioning(RF) and emotional functioning has got also difference but not significant statistically (0.061 and 0.070).
Table IV: showing Breast cancer patients' pre-diagnosis and follow-ups functioning and symptoms scores as measured by the EORTC QLQ - BR23* $(n=250)$

\begin{tabular}{lccccc}
\hline Parameters & $\begin{array}{l}\text { Mean } \\
\text { score } \\
\text { (M1) }\end{array}$ & SD & $\begin{array}{l}\text { Mean } \\
\text { score } \\
\text { (M2) }\end{array}$ & SD & $\mathrm{p}$ \\
\hline $\begin{array}{l}\text { Body } \\
\text { image(BRB1) } \\
\text { Sexual } \\
\text { functioning } \\
\text { (BRSEF) }\end{array}$ & 86.00 & 7.83 & 70.46 & 6.36 & .041 \\
$\begin{array}{l}\text { Sexual } \\
\text { enjoyment } \\
\text { (BRSEE) }\end{array}$ & 83.47 & 7.55 & 72.05 & 2.33 & .015 \\
$\begin{array}{l}\text { Future } \\
\text { Perspectives } \\
\text { (BRFU) }\end{array}$ & 75.62 & 7.49 & 62.53 & 11.17 & .08 \\
$\begin{array}{l}\text { Breast } \\
\text { symptoms } \\
\text { (BRBS) }\end{array}$ & 98,82 & 1.66 & 91.39 & 3.38 & .017 \\
$\begin{array}{l}\text { Arm } \\
\text { Symptoms } \\
\text { (BRAS) }\end{array}$ & 95.03 & 7.02 & 77.60 & 3.11 & .018 \\
$\begin{array}{l}\text { Upset by hair } \\
\text { loss(BRHL) }\end{array}$ & 74.98 & 35.38 & 64.30 & 40.87 & .269 \\
\hline
\end{tabular}

*Scores range from 0 to 100 , with higher scores representing a higher level of symptoms

Other features like emotional functioning (EF) and cognitive functioning, (CF) though they have significant value $(\mathrm{p}=0.001$ and .01$)$ in the premastectomy phase, in the post mastectomy phase they had no much significance $(p=0.07$ and 0.06). All the general features deteriorated after mastectomy. Other features like presence of fatigability, nausea, apatite loss, sleeplessness and pain has got tremendous effect on the quality of life. On the second point pain and fatigability was remarkable $(\mathrm{p}=0.05)$. Pain score was lower (59.21/54.01) in the second point. Apatite loss and dyspnea increased after surgery (Table II and III). Breast cancer symptoms: Body image (BRB1), Sexual functioning (BRSEF), Sexual enjoyment (BRSEE), Future Perspectives (BRFU), Breast symptoms (BRBS), Arm Symptoms (BRAS) Table IV. After diagnosis the patient bear tremendous moral strength in respect to their body image (BRB1) $\mathrm{p}=0.041$, future sexual functioning and future perspectives $(\mathrm{p}=0.023,0.045)$ with minimum arm symptoms $(\mathrm{p}=0.033)$. On the second point after surgery, they lose tremendous effect on this parameter particularly on the body image, breast and arm symptoms (86.00/70.46, $98.82 / 91.39,95.03 / 77.07)$ respectively on two points, $\mathrm{p}$ values are $0.013,0.017$ and 0.018 . Patients did have much effect on sexual 
functioning ( $\mathrm{p}=0.015)$, but they lost much effect on the future perspectives also (75.62/62.53), $\mathrm{p}=$ 0.080 .

\section{Discussion}

This study provided data on health-related quality of life of 250 breast cancer patients and measures immediate impact on the QOL after mastectomy by two-point interview and examination using standard quality of life measures. Mean age is 44.7 which is much lower than that of many series though incidence increases with age up to 50 years. The cumulative incidence of breast cancer among women in Europe and North America is about $2.7 \%$ by age 55 , about $5.0 \%$ by age 65 , and about $7.7 \%$ by age $75 .{ }^{14}$

The purpose of our study was, first, to investigate changes of QOL in breast cancer patients after mastectomy and, second, to examine differences of QOL in oncological subgroups. The QLQ-C30, QLQ-BR23 were used as valid, reliable, and useful clinical measures. ${ }^{15}$ In our analysis, a $\mathrm{p}$ value $\leq 0.05$ was chosen to test for changes in the whole sample; however, no adjustments for multiple testing were made.

Most QOL results changed over time: especially there is a difference of it before and after operation particularly after mastectomy. In this series, all components of QOL like physical functioning, emotional functioning, role functioning, cognitive functioning deteriorated, on the other hand sleeping status, social functioning and symptoms improved after surgery. These pictures vary in different studies. ${ }^{16}$

Regarding breast cancer scores show many of the parameters deteriorated after surgery especially arm symptoms. There are mentionable effects of neoadjuvant chemotherapy on the QOl. On the whole, results confirm that QOL in breast cancer patients improved over time, but there were still impairments regarding anxiety, body image, and sexual functioning. Younger patients were much more distressed by cancer diagnosis and treatment. However, at the time of cancer diagnosis, and as well as later on, surgical modality and tumor prognostic factors seemed to play a minor role in patients' subjective QOL., which can be discussed in terms of the "well-being Decreased cognitive functioning was observed at three months' assessment that continued to be persistent at 18 months' follow-up. Long-term (1 to 10 years) cognitive impairment in patients with breast cancer after their chemotherapy treatment has been reported. ${ }^{17}$

Except for future perspective all the other breast cancer specific functioning including body image, sexual functioning and sexual enjoyment decreased at 18 months' follow-up assessment. Sexual dysfunction is a symptom that may occur as a result of premature menopause following adjuvant systemic therapy in breast cancer patients. ${ }^{15}$ In this study we faced difficulties in eliciting response to sex related questions as majority of our patients are illiterate and conservative feels shy to interact in this part of their life.

Most patients in this study were diagnosed with advanced disease (loco-regional 53.6\%). A study on cancer practice by general surgeons in Bangladesh showed that surgeons do not routinely perform breast conserving surgery as the first treatment modality for breast cancer patients. It needs further attention in order to improve both early diagnosis and clinical outcomes. However, this study was limited due to its small cohort of breast cancer patients. In addition, patients were relatively young, although studies have shown that in Bangladesh breast cancer patients present with advanced stage and they are about more than 10 years younger than their western counterparts. Furthermore, most of the functional scores did not improve over time and this is in contradiction to the findings from some existing literature. ${ }^{10}$

We found that, at the end of treatment, women reported a normal level of mental health, with little evidence of being depressed or having negative effect, despite prolonged and complex treatments. However, they reported a broad range of physical symptoms, including hot flashes, night sweats, aches and pains, and vaginal dryness. By contrast, women, especially those who had a mastectomy, reported physical functioning scores at the end of primary treatment that were much lower consistent with other research on sexual functioning after breast cancer, more sexual problems, specifically difficulties with sexual interest, lubrication, and pain with intercourse, were reported by patients who received adjuvant chemotherapy than by patients who received surgery only. ${ }^{18-20}$ 
Moving Beyond Cancer: The movement between health and illness involves passages of both change and adaptation. Though bodily changes such as hair and breast loss. For many women, an altered body appearance resulted in a disruption to identity and sense of self and mental agony. Following surgery for breast cancer, women often have to follow a course of chemotherapy or hormonal therapy to reduce the chances of cancer recurrence. The movement from health to illness signifies a range of psychological processes involved in adapting to this changed event or the disruption that has occurred. ${ }^{21}$

Such adaptation is a continual and uncertain process for women during their movement through the breast cancer experience. Indeed, the movement from health to illness signifies adaptation in addition to change. The completion of active treatment and stages in the journey towards living with cancer also result in multiple transitional processes and experiences for many women diagnosed with the disease. Difficulties moving on from active treatment can be exacerbated because of the range of changes to women's bodies. Many of these act as a constant reminder of the illness and have consequential limitations and disabilities extending beyond treatment. $^{22}$

At the end of primary treatment for breast cancer, women in this study reported decreased energy and many treatment-associated symptoms, accounting for the substantial decreases in physical, emotional and cognitive functioning. However, despite these symptoms, women's emotional functioning was generally in the normal range for healthy women, with little evidence of depressed mood or negative affect. Although this finding was reassuring and contrary to what had been expected from review of the literature, group mean data may mask the experience of those individual women with breast cancer who experience more emotional distress than average and may be less resilient in responding to the diagnosis and treatment than other women with this disease. ${ }^{23}$ According to results showed by QLQ C30, women of age > 40 years have better QOL than the younger group which is not consistent with other studies, most of the subjects were housewives, illiterate and unemployed which is not seen in other studies. ${ }^{24}$
Global health status QOL had found significant relationship $(\mathrm{p}<0.05)$ with emotional functioning, dyspnea, body image and inverse relationship $(p<0.05)$ with breast symptoms. Arm symptoms and upset by hairless the results are consistent with other study. In multivariate regression analysis body image, future perspectives and breast symptoms had significant impact on GHS QOL ( $\mathrm{p}<.05)$. A recent study in Japan shows that Breast Conservation Surgery (BCS) results in better QoL or body image, and this can be the foundation for actively advocating BCS among patients with breast cancer. In the meantime, for those who have undergone Total Mastectomy, emotional support and management of symptoms will need to be actively provided through consultation, education and rehabilitation. Although there were a few differences on some scales, patients who underwent total mastectomy had a similar QoL. Efforts to evaluate and improve QoL of the patients with breast cancer should be continued. ${ }^{25}$

Conclusion: A diagnosis of cancer and associated treatments affects multiple domains of life and consequentially immerses patients into a complex web of interrelated experiences. Furthermore, the complicated transitions between health, illness and living with cancer can often be compounded by challenges and threats to one's identity and self. Though this study only covers effects of women after mastectomy, there might have change during and after adjuvant therapies. So it needs details of MBC study.

A preoperative information for the patient confronted with breast cancer should include possible psychological effects of cancer diagnosis, surgery, and other treatment. Patients should be informed that impairments of QoL are likely to occur, but will decrease over time.

The patient should be encouraged to communicate her problems and seek professional help. Because of greater distress and demands in family and joblife after treatment, special psycho-oncological groups for younger patients might be useful. Screening measures could help to evaluate the individual need for psycho-oncological treatment. Now a day with the improvement of education and economy, on the basis of patients' demand breast conservative treatment, different oncoloplastic surgeries might alleviate the sufferings and distresses due to unwanted body image. 


\section{Acknowledgement}

We could carry out this project by a liberal fund from Bangladesh Medical Research Council. So many thanks to BMRC authority. Special thanks to the administrative authority, staffs of different categories of the department of surgical oncology of National Institute of Cancer Research \& Hospital, Dhaka for their kind support and cooperation to finish this tedious job.

\section{References}

1. Ries LAG, Eisner MP, Kosary CL, et al. (eds): SEER Cancer Statistics Review, 1975-2000. Bethesda, MD, National Cancer Institute,2003. http://seer.cancer.gov/csr/1975_2000.

2. Mullan F: Re-entry: The educational needs of the cancer survivor. Health EducQ10:88-94,1984 (suppl)

3. Mullan F: Seasons of survival: Reflections of a physician with cancer. N Engl J Med 1985; 313: 27073.

4. cKinley ED: Under toad days: Surviving the uncertainty of cancer recurrence. Ann Intern Med 133:479-80, 2000.

5. Degner LF, Kristjanson LJ, Bowman D, Sloan JA, Carriere KC, O'Neil J, et al.: Information needs and decisional preferences in women with breast cancer. JAMA 1997; 277: 1485-92.

6. Burstein HJ, Winer EP. Primary care for survivors of breast cancer. N Engl J Med 2000; 343: 1086-94.

7. Ganz PA. Impact of quality of life outcomes on clinical practice. Oncology. 1995; 9:61-65.

8. Hartl K, Rebecca S. Marianne M., Jutta E. Hans R., Harald S. Klaus F. Quality of Life, Anxiety, and Oncological Factors: A Follow-Up Study of Breast Cancer Patients. Psychosomatics 2010; 51:112-23.

9. Stewart BW, Paul Kleihues P: World Cancer Report. Lyon, France, International Agency Research on Cancer; 2003.

10. Montazeri A. Health-related quality of life in breast cancer patients: A bibliographic review of the literature from 1974 to 2007. J Experim Clin Cancer Res 2008, 27:32.

11. Eifel P, Axelson JA, Costa J, Crowley J, Curran WJ $\mathrm{Jr}$, Deshler A, et al. National Institutes of Health Consensus Development Conference Statement: adjuvant therapy for breast cancer, November 1-3, 2000. J Natl Cancer Inst 2001; 93:979-89.
12. National Center for Health Statistics CDCP. US Mortality Public Use Data Tapes, 1969-2003. 2006.

13. American Cancer Society ISDH., Indiana Cancer Consortium Indiana Cancer Facts and Figures 2006: A sourcebook for planning and implementing programs for cancer prevention and control. 2006.

14. Collaborative Group on Hormonal Factors in Breast Cancer. Breast cancer and hormone replacement therapy: collaborative reanalysis of data from 51 epidemiological studies of 52705 women with breast cancer and 108411 women without breast cancer. Lancet 1997; 350: 1047-59.

15. Hartl K, Ph.D., Schennach R, Muller M, M.D., Ph.D., Engel J, M.D., Reinecker H, M.P.H., Ph.D., Sommer H, M.D., Friese K, M.D., Ph.D.: Quality of Life, Anxiety, and Oncological Factors: A Follow-Up Study of Breast Cancer Patients. Psychosomatics 2010; 51:112-23.

16. Mehnert A, Koch U: Prevalence of acute and posttraumatic stress disorder and comorbid mental disorders in breast cancer patients during primary cancer care: a prospective study. Psycho-Oncology $2007 ; 16: 181-88$.

17. Millar K, Purushotham AD, McLatchie E, et al: A 1year prospective study of individual variation in distress, and illness perceptions, after treatment for breast cancer. J Psychosom Res 2005; 58:335-42.

18. Ganz PA, Desmond KA, Belin TR, Meyerowitz BE, Rowland JH. Predictors of sexual health in women after a breast cancer diagnosis. J Clin Oncol 1999; 17:2371-80.

19. Berglund G, Nystedt M, Bolund C, Sjoden PO, Rutquist LE. Effect of endocrine treatment on sexuality in premenopausal breast cancer patients: a prospective randomized study. J Clin Oncol 2001; 19:2788-89.

20. Brezden CB, Phillips KA, Abdolell M, Bunston T, Tannock IF. Cognitive function in breast cancer patients receiving adjuvant chemotherapy. J Clin Oncol 2000;18: 2695-701.

23. Kralik D, Visentin K \& van Loon A. Transition: a literature review. J Advan Nurs 2006; 55, 320-29.

24. McCann L, Illingworth N, Wengstro'm Y, Hubbard G, and Kearney N. Transitional experiences of women with breast cancer within the first year following diagnosis. J Clin Nurs 2010; 19: 1969-76.

25. Ganz PA, Kwan L, Stanton AL, Krupnick JL, Rowland JH, Beth E, et al. Quality of Life at the End of Primary Treatment of Breast Cancer: First Results from the Moving Beyond Cancer Randomized Trial.J Nat Cancer Inst. 2003; 96: 376-87 